\title{
MULTI-FREQUENCY SECOND HARMONIC GENERATION IN IRREGULAR TWO DIMENSIONAL APERIODIC OPTICAL SUPERLATTICES
}

\author{
N. SANG NOUR POUR * \\ Department of Theoretical Physics, Faculty of Physics, University of Tabriz, Tabriz, 51664, Iran \\ Research Institute for Applied Physics and Astronomy, University of Tabriz, Tabriz, Iran \\ n.sangnoor@gmail.com

\section{NEMATI} \\ Department of Theoretical Physics, Faculty of Physics, University of Tabriz, Tabriz, 51664, Iran \\ Research Institute for Applied Physics and Astronomy, University of Tabriz, Tabriz, Iran \\ Yerevan State University, Alex Manoogian 1,0025, Yerevan, Armenia \\ m.nemati55@gmail.com

\section{GOALIPOUR} \\ Department of Theoretical Physics, Faculty of Physics, University of Tabriz, Tabriz, 51664, Iran \\ golipormehdi@yahoo.com
}

\section{REZAEI}

Department of Theoretical Physics, Faculty of Physics, University of Tabriz, Tabriz, 51664, Iran Department of Physics, Applied science faculty, Simon Fraser University, Burnaby, Canada karamaty@tabrizu.ac.ir

\section{R. KHERADMAND}

Research Institute for Applied Physics and Astronomy, University of Tabriz, Tabriz, Iran r_kheradmand@tabrizu.ac.ir

\section{G. YU. KRYUCHKYAN}

Yerevan State University, Alex Manoogian 1, 0025, Yerevan, Armenia Institute for Physical Researches, National Academy of Sciences Ashtarak-2, 0203 Ashtarak, Armenia kruchkyan@ysu.am

\begin{abstract}
We investigate two dimensional nonlinear aperiodic structures leading to multi quasi-phasematching conditions and multi-frequency second-harmonic generation (SHG) in one chip. For arranging devices close to an experimental situation the intensities of SHG in dependence from frequencies and direction of radiation are studied for irregular structures with random fluctuations of the sizes of each poling domain. Comparing the regular and irregular two dimensional nonlinear aperiodic structures we study the role of imperfection effects of random fluctuations in multi-frequency SHG.
\end{abstract}

Keywords: Nonlinear Optics; Harmonic generation and multiple frequency process; Quasi-phasematching. 


\section{Introduction}

Nonlinear interactions have been used for production of new frequency laser sources. One of the important applications of second-order nonlinear interactions is second harmonic generation (SHG). It is well known that efficient SHG and the other threewave parametric processes depend critically on the phase-matching conditions usually achieved through the crystal birefringence or quasi-phase-matching (QPM) ${ }^{1}$. The last method based on periodic modulation of the second order nonlinear susceptibility is naturally realized in periodically poled nonlinear crystals (PPNC) in which the reciprocal wave vectors in media are used to compensate mismatch between interacting waves in nonlinear process. QPM is usually realized for collinear one-dimensional propagation of the fundamental and second-harmonic waves along the reciprocal vector. An important extension of one-dimensional gratings is engineering of nonlinear crystals with twodimensional (2D) modulation of the sign of the second-order susceptibility ${ }^{2-4}$. Periodic grating of the second order nonlinear susceptibility, either in one dimension or in two dimensions is widely used in the last time for QPM processes including SHG. Many kinds of 2D structures has been proposed that lead to the generation of different kinds of the second-harmonic ( $\mathrm{SH}$ ) scattered patterns ( $\mathrm{see}^{5}$, and Refs. therein).

In recent years, there has been a growing interest in elaboration of multi-frequency SHG in which the phase-matching can be simultaneously satisfied by a number of different wave lengths. These kinds of processes are noticeable in different applications including optical information processing and telecommunication as well as various medical sensing applications and agrichemical analyses ${ }^{6}$. For this purpose, different configurations such as Fibonacci structure and aperiodic optical super-lattices (AOS) are recently offered ${ }^{7-10}$. Multi-QPM condition in AOS structures is used to create multi-frequency (MF) in which QPM condition is satisfied with the suitable definition of reciprocal vectors such that supply even more reciprocal vectors for multi-QPM in one and two dimension ${ }^{7,11}$. The AOS structure is made of random modulation of the sign of second order nonlinear coefficient of domains in the layered structure such that the length of each domain is an integer multiple of a unit block. Simulation and optimization methods are used to the design of AOS structures, one of these methods is Simulated Annealing (SA) which is used in the design optimal one and two dimensional crystals which are recently achieved $^{8}$. The various structures of PPNC and combinations of linear-nonlinear AOS layered structures in SHG and down-conversion are studied in the papers ${ }^{12-18}$. These studies show that the output efficiency of SHG in AOS structure for MF is considerable.

In the present paper the multi-frequency SHG satisfying multi-QPM conditions in two-dimensional aperiodic poled layered structures are studied. The novelty is that effects of fluctuations in the lengths of each domain leading to irregularity in AOS structures are investigated. The obtained results are compared with the analogous results for the regular aperiodic poled structure. Therefore, the investigation of the optimization design method for constructing AOS with the help of SA method in two-dimension is suggested. At first, a definition of the AOS structure in the real space representation is presented. Then a comparison between SHG in regular and irregular structures is done. 
The paper is constructed as follows: in Sec. 2 the regular structure for SHG in twodimensions is explained and SA method for obtaining an optimal AOS structure in twodimension is considered. Next, in Sec. 3 the results are discussed by considering the behavior of the effective nonlinear coefficient with respect to wavelength in the regular and irregular models. We summarize our results in Sec. 4 .

\section{Method numerical simulation for AOS}

We consider a two-dimensional nonlinear aperiodic structure which is based on ferroelectric crystals, e.g., $\mathrm{LiNbO}_{3}$ and $\mathrm{LiTaO}_{3}$ in which the sign of the susceptibility may be locally inverted by a domain reverse. Our goal is engineering the structures which are proper for conversion of one or more frequencies in the experimental situation is needed. The performance of these kinds of processes cause to several amplified SH frequencies which are called MF conversion. In order to having a pattern with high efficiency of emerged photons the appropriate arrangement of the positive and negative domains is needed which is the result of optimization of the construction.

We follow the practical method proposed for consideration the random orientations of second order susceptibility in PPNC structure ${ }^{7}$. In this method, the sign of the second order nonlinear susceptibility is aperiodically inverted through the crystal (Figs. 1). The idea of 2D-AOS in the SHG process is represented in Figs. 1(a) and 1(b). In Fig. 1(a) the AOS in multi-SHG process is represented which black-white area are related to positivenegative second order susceptibility, respectively. Fig. 1(b) represents the wave vectors of fundamental and $\mathrm{SH}$ waves where $\theta$ is the angle between the incidents and output wave vectors, while $\vec{G}$ is the reciprocal lattice vector.
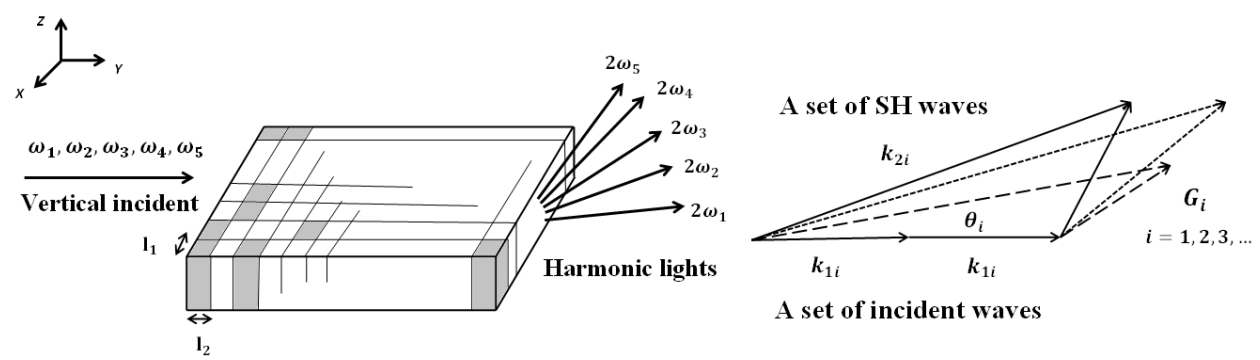

A set of incident waves

Fig.1. The designed structure for 2D-AOS with $\chi^{+}>0$ and $\chi^{-}<0$. The domain orientation is represented by the poling function $\mathrm{g}(\mathrm{x}, \mathrm{y})$ which is determined by the method of simulated annealing (SA). The diagram of reciprocal lattice vectors in multi-frequency SHG process in momentum space.

We consider SHG of a set of laser beams with frequencies $\omega_{1}, \omega_{2}, \omega_{3}, \omega_{4}, \omega_{5}$ propagated in the $x-y$ plane through AOS. In the assumption of slowing-wave variation of the field amplitude the conversion efficiency of the output signal can be presented as below:

$$
\eta=\frac{I_{2 \omega}}{I_{\omega}}=\frac{8 \pi^{2}\left|d_{33}\right|^{2} I_{\omega} S^{2}}{c \varepsilon_{0} n_{\omega}{ }^{2} n_{2 \omega} \lambda^{2}} d^{2},
$$


where $d_{33}$ is the nonlinear susceptibility of the sample, $I_{\omega}$ and $I_{2 \omega}$ are the input and output intensities of the process respectively, $\lambda$ is the wavelength of input wave and $n_{\omega}$ and $n_{2 \omega}$ are refractive indexes for fundamental and SH waves respectively. We denote here the second order nonlinear coefficient as:

$$
d=\left|\frac{1}{S} \iint_{S} g(x, y) e^{-i\left(\vec{k}_{2 \omega}-2 \vec{k}_{\omega}\right) \cdot \vec{r}} d x d y\right|,
$$

where $S$ is the area of the two-dimensional periodic structure and $k_{\omega}$ and $k_{2 \omega}$ are the wave vectors for fundamental and SH waves respectively, and $r$ is the position vector in the sample. We rewrite this expression for the layered structure including additionally the poling function $g(x, y)$ that takes values of $(1$ or -1$)$ for the original and inverted polarization of domains, respectively. For a discrete layered media the poling function can be written as $g(n, m)$ for the ranges of positive-and negative-susceptibilities (see, Figs. 1) in the ranges $x \in\left[n l_{1}, n l_{1}+l_{1}\right], y \in\left[m l_{2}, m l_{2}+l_{2}\right]$ under the division above of the surface. In this case, as a result, the modified effective nonlinear coefficient can be expressed as below:

$$
d_{e f f}=\frac{d_{33}}{N_{1} N_{2}}\left|\sin c(u) \sin c(v) \sum_{n m} g(n, m) e^{-i((2 m+1) u+(2 n+1) v)}\right| .
$$

where $G_{x}=k_{2} \cos (\theta)-2 k_{1}, G_{y}=k_{2} \sin (\theta), r$ is the position vector in $x$-y plane and $\sin c(x)=\sin (x) / x$. The functions $u$ and $v$ are defined as $u=l_{1} G_{x} / 2, v=l_{2} G_{y} / 2 . N_{1}$ and $N_{2}$ are the number of domains, and $l_{1}$ and $l_{2}$ are the length of domains, in $x$ and $y$ direction, respectively.

The idea which is used in the design of these grating approaches is that introducing the poling function and several parameters into the Fourier transform of second-order susceptibility, we then adjust these parameters for obtaining strong Fourier coefficients at the required phase matching wave vectors. According to Eq. 1, the output efficiency is proportional to $d_{\text {eff }}{ }^{2}$ thus, the optimal construction in aperiodic structure can be described as a method for finding several maximum values of $d_{\text {eff }}$ in SHG. The phase mismatch problem in PPNC is solved with the domain orientation between the two adjust domains and the ideal constructive interference emerges. But in aperiodic structure the condition is complicated and depends on solving an inverse source problem ${ }^{7}$. These kinds of nonlinear optimization problems require using mathematical optimization procedures over the output waves to have desired rations of the output intensities of SH waves. One of the useful practical algorithms in this case is Simulated Annealing ${ }^{19}$ which is a powerful method for finding the global extremum of an objective function that may include local minimum. The objective function " $F$ " is considered which should be minimized to have the favorable arrangement of orientation of layers in the crystal. This function in the SA method is considered as follow:

$$
F=\sum_{i}\left|d_{\text {eff }}\left(\lambda_{i}\right)\right|+A\left\{\max \left(d_{\text {eff }}\left(\lambda_{i}\right)\right)-\min \left(d_{\text {eff }}\left(\lambda_{i}\right)\right)\right\} .
$$


Here the maximum and minimum values among all the quantities included in the parenthesis is represented with the symbols max and min and $A$ is a constant to balance each $d_{\text {eff }}$. As an example, five wavelengths of $1.4 \mu \mathrm{m}, 1.45 \mu \mathrm{m}, 1.5 \mu \mathrm{m}, 1.55 \mu \mathrm{m}$ and $1.6 \mu \mathrm{m}$ of the fundamental wave and the output angles of $\theta=10, \theta=11, \theta=12, \theta=13$ and $\theta=14$ degrees are chosen. In this construction, the length of segments are $l_{1}=l_{2}=1 \mu \mathrm{m}$, the number of layers $N_{1}=N_{2}=100$ is considered in order to carry out the particular construction of two dimensional AOS in which the above SHG process at the five wavelengths and angles performs. The dispersion of the refractive index of the material is evaluated through Sellmeier formula which is calculated at the room temperature for the fundamental and SHG wavelengths ${ }^{20}$.

\section{Results and discussion}

Utilizing 2-dimensional AOS (2D-AOS) for the SHG process, we study in details the behavior of $d_{\text {eff }}$ in both cases of the regular and irregular 2-dimensional grating with respect to the various properties of the structure. For this purpose, the structure is considered to determine the effect of fluctuation in the length of each domain in $x$ and $y$ directions on the $d_{\text {eff }}$. The results of numerical simulations by SA method, for the efficiency of the multiple SHG, through the effective nonlinear coefficient are represented in Figs. 2 and 3.

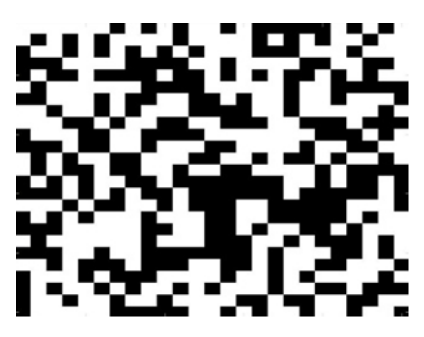

(a)

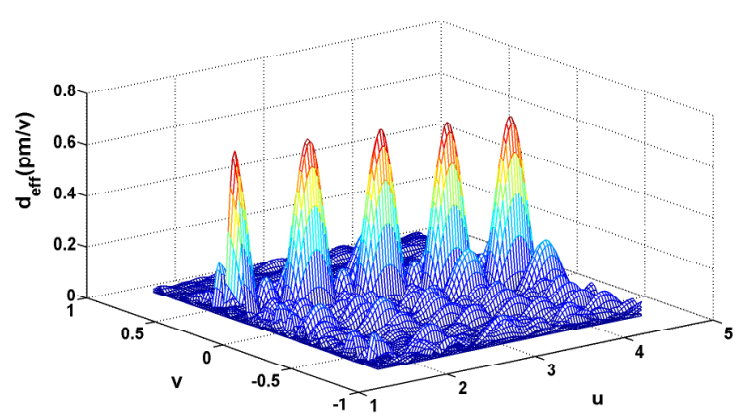

(b)

Figs. 2. (a) the typical gray scale diagram of the constructed 2D-AOS, the black (white) segments illustrate the positive (negative) susceptibilities in the domains. (b) The behavior of the reduced effective nonlinear coefficient at the five given fundamental wavelengths with respect to $u$ and $v$ in the perfect 2D-AOS.

Parameters $\mathrm{u}$ and $\mathrm{v}$ are related to reciprocal vectors in 2 dimensional grating.

Fig. 2(a) represents the gray scale diagram of the constructed 2D-AOS, where the black (white) segments show the positive (negative) second order susceptibilities and Fig. 2(b) represents the computed $d_{\text {eff }}$ in optimized 2D-AOS after scanning a various amounts of $G_{\mathrm{x}}$ and $G_{\mathrm{y}}$. Five maximum peaks with almost identical value of about 0.672 at the fundamental wavelengths and output angles are achieved. 
Now, we consider the role of length fluctuations on each layer of the 2D-AOS which is obtained by using SA method. The behavior of $d_{\text {eff }}$ for irregular structures is presented in the Figs. 3 (a, b). For consideration of the role of fluctuation in the length of each domain and attain its effect on $d_{\text {eff }}$ we modify the simulation method. For this purpose, we take into account a Gaussian random number generator (between 0 and 1 with equal probability) to consider all possible values of fluctuation in the length of segments (the length of each segment may rise or fall from the designed length). Therefore, we considered the cosine of the output of the random generator multiplied by $2 \pi$ and then multiply the output by a constant scaling coefficient which determines the maximum value of fluctuations in the length of blocks. The coefficient of fluctuations in the lengths of each layer in the structure in Figs. $3(\mathrm{a}, \mathrm{b})$ are $0.02 \times \cos (2 \pi \times R)$, and $0.05 \times \cos (2 \pi \times R)$ respectively ( $R$ is a Gaussian random number).

Figs. 3-(a) and 3-(b) illustrate the computed $d_{\text {eff }}$ for the optimized irregular 2D-AOS with respect to $u$ and $v$ parameters. The maximum value of fluctuation in Figs. 3-(a) and 3 -(b) are 2 and 5 percent of the designed length of each segment respectively. As it can be seen from the figures, various fluctuations in the length of segments in the same structure illustrate different results. It is evident that as the amount of fluctuation rises, the maximum value of $d_{\text {eff }}$ decreases. Paying attention to Fig. 3-(b) reveals that increasing the percentage of fluctuation leads to a rapidly decreasing behavior of the $d_{\text {eff }}$. Therefore, it is obvious that as the amount of fluctuation increases the regular behavior of the output efficiency disappears such that the value of achieved peaks in five predesigned wavelengths decreases.

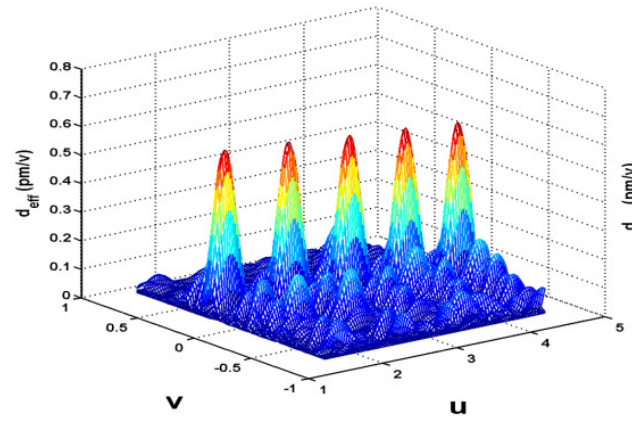

(a)

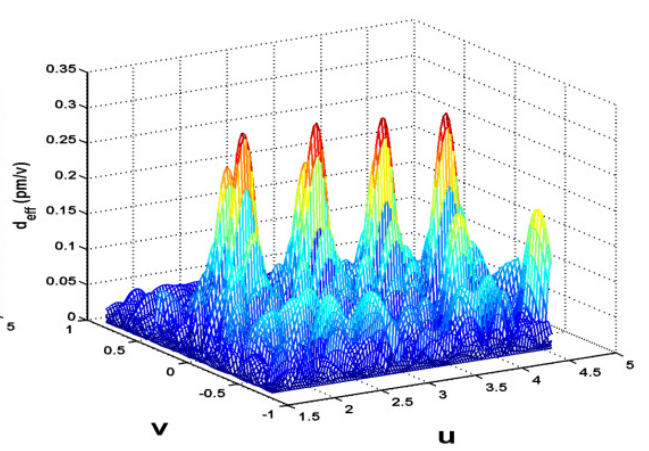

(b)

Fig.3. The behavior of the reduced effective nonlinear coefficient for MF SHG in irregular AOS structure. (a) assumed maximum amount of fluctuation is $2 \%$ of the length of each pre-designed domain; (b) assumed maximum amount of fluctuation is $5 \%$ of the length of each pre-designed domain. Parameters $\mathrm{u}$ and $\mathrm{v}$ are related to reciprocal vectors in 2 dimensional grating.

\section{Conclusion}

In summary, we have investigated two-dimensional aperiodic optical grating for multifrequency SHG process on the base of numerical simulation. The effect of random 
fluctuations in the lengths of each domain has been analyzed for efficiency of multiple SHG. Comparing the results for regular and irregular AOS configurations it has been demonstrated that the intensities of multi-frequency SHG are very sensitive to random fluctuations of the borders of polling domains. The moderate percentage of fluctuations leads to irregular behavior of SHG for pre-designed wavelengths.

\section{References}

1. M. M. Fejer, G. A. Magel, D. H. Jundt and R. L. Byer, IEEE J. Quant. Electron. 28, 2631 (1992).

2. V. Berger, Phys. Rev. Lett. 81, 4136 (1998).

3. N. G. R. Broderick, G. W. Ross, H. L. Offerhaus, D. J. Richardson and D. C. Hanna, Phys. Rev. Lett. 84, 4345 (2000).

4. S. M. Saltiel, A. A. Sukhorukov and Y. S. Kivshar, Prog. Optics 47, 1-73 (2005).

5. A. Arie, N. Habshoosh and A. Bahabad, Opt. Quant. Electron. 39, 361 (2007).

6. S. N. Son, J. J. Song, J. U. Kang and C. S. Kim, Sensors 11, 6125 (2011).

7. B. Y. Gu, Y. Zhang and B. Z. Dong, J. Appl. Phys. 87, 7629 (2000).

8. H. Liu, S. N. Zhu, Y. Y. Zhu, N. B. Ming, X. C. Lin, W. J. Ling, A. Y. Yao and Z. Y. Xu, Appl. Phys. Lett. 81, 3326 (2002).

9. L. M. Zhao, B. Y. Gu, Y. S. Zhou and F. H. Wang, J. Appl. Phys. 94, 1882 (2003).

10. L. M. Zhao, B. Y. Gu, Y. S. Zhou and F. H. Wang, J. Phys.: Condens. Matter. 15, 4889 (2003).

11. H. Liu, Y. Y. Zhu, S. N. Zhu, C. Zhang and N. B. Ming, Appl. Phys. Lett. 79, 728 (2001).

12. D. A. Antonosyan and G. Yu. Kryuchkyan, "Multiple Interactions in Multilayered Structures of Nonlinear Materials", Modern Optics and Photonics: Atoms and Structured Media. Eds: G. Kryuchkyan, G. Gurzadyan and A. Papoyan, World Scientific, 131-150, (2010).

13. D. A. Antonosyan, T. V. Gevorgyan, and G. Yu. Kryuchkyan, Phys. Rev. A 83, 043807 (2011).

14. D. A. Antonosyan and G. Yu. Kryuchkyan, Opt. Comm. 285, 795 (2012).

15. M. Rezaei, R. Kheradmand, G. Yu. Kryuchkyan and M. Nemati, J. Nonlinear Optical Physics \& Materials. 20, 23 (2011).

16. R. Kheradmand, M. Rezaei, G. Yu. Kryuchkyan, M. Nemati and A. Razmi, Phys. Status. Solidi A. 208, 1849 (2011).

17. D. A. Antonosyana, M. Nemati and G. Yu. Kryuchkyana, Proc. of SPIE 8414, 84140T-1 (2012).

18. D.A. Antonosyan, T.V. Gevorgyan and G.Yu. Kryuchkyan, Proc. of SPIE 7998, 799816-1 (2011).

19. S.Kirkpatrick, C. D. Gelat and M. P. Vecchi, Science 220, 671 (1983).

20. J. P. Meyn and M. M. Fejer, Opt. Lett. 22, 1214 (1997). 